\title{
Congenital Glaucoma-A Brief Review
}

\author{
R Krishnadas, R Ramakrishnan
}

Aravind Eye Care System, Tamil Nadu, India

\section{NOMENCLATURE AND CLASSIFICATION}

Congenital glaucomas are present at birth while infantile glaucomas refer to elevated ocular pressures and other sequelae with onset from birth up to three years of life. Developmental glaucomas are associated with developmental anomalies of the eye present at birth. Defective primary development of the outflow structures of the eye causes primary developmental glaucomas whereas other developmental ocular abnormalities secondarily affecting the aqueous outflow structures cause secondary developmental glaucomas (e.g. glaucoma secondary to persistent primary hyperplastic vitreous, spherophakia, retinopathy of prematurity, Sturge Weber syndrome, congenital ectropion uveae, etc). This review will focus on the most common form of developmental glaucomas seen in ophthalmic practice, Primary Congenital Glaucoma. The reader is referred to standard textbooks on glaucoma for a detailed description of the terminology and classification of developmental glaucomas. Buphthalmos is derived form the Greek term for ox-eye and refers to the significant enlargement of the eye that can occur as a consequence of any type of glaucoma present in infancy. Similarly, Hydrophthalmos in Greek refers to the high fluid content present with marked enlargement of the eye. These are merely descriptive terms with no implications on the etiology, pathogenesis or management of the eyes so affected, and are not used diagnostically in clinical practice.

Primary congenital glaucoma (commonly, alternately and widely referred to as primary infantile glaucoma as well) is described by Shaffer and Weiss ${ }^{1}$ as follows:

Primary congenital glaucoma is the most common hereditary glaucoma of childhood, inherited as an autosomal recessive pattern, with specific angle anomaly consisting of absence of angle recess with iris insertion directly into trabecular surface. There are no other development anomalies of the eye. Elevated IOP results in corneal enlargement, clouding and breaks in Descemet's membrane.

\section{Pathophysiology of Primary Congenital Glaucoma}

IOP elevation in primary congenital glaucoma is due to an abnormal development of the anterior chamber angle that results in reduction of aqueous outflow facility and there is no uniform agreement among investigators on the nature of obstruction to aqueous flow in congenital glaucoma. Barkan² suggested incomplete resorption of mesodermal tissue led to formation of a membrane across the anterior chamber angle, referred to as the Barkan's membrane and forms the basis of the surgical procedure of goniotomy which results in cleaving of the membrane to increase aqueous flow. The existence of such a membrane has not been proved by light or electron microscopy. Maumenee ${ }^{3}$ demonstrated abnormal anterior insertion of ciliary muscle over the scleral spur in eyes with infantile glaucoma. He observed that longitudinal and circular fibers of the ciliary muscles inserted directly onto the trabecular meshwork rather than the scleral spur and root of the iris inserts directly to trabecular meshwork. Anderson ${ }^{4}$ provided histopathological support for the high insertion of iris into trabecualr meshwork, suggesting this is due to a development arrest in the normal migration of anterior uvea across the meshwork in the third trimester of gestation. The eyes in primary congenital glaucoma are characterized by the appearance of the iris and ciliary body in the seventh of eighth month of gestation rather than the full term development of the infant. The iris and ciliary body have failed to recede posteriorly with resultant overlap of the iris insertion and anterior ciliary body over the posterior trabecular meshwork (Fig. 1). It has also been postulated that abnormal extracellular matrix and glycoproteins cause abnormal anterior segment development.

Primary congenital glaucoma appears to result from developmental anomaly of the anterior segment structures derived from the embryonic neural crest cells causing outflow obstruction to aqueous by several mechanisms. Developmental arrest may result in anterior insertion of iris, direct insertion of the ciliary body onto the trabecular meshwork and poor structural development of the scleral spur. The high insertion of the ciliary body and the iris onto posterior trabecualr meshwork may compress the trabecualr beams and the extracellular meshwork may be abnormal.

\section{Epidemiology and Genetics}

Primary congenital glaucoma is an extremely rare inherited eye disease and accounts for $0.01-0.04 \%$ of total blindness and is the most common form of glaucoma in children. The disease is 

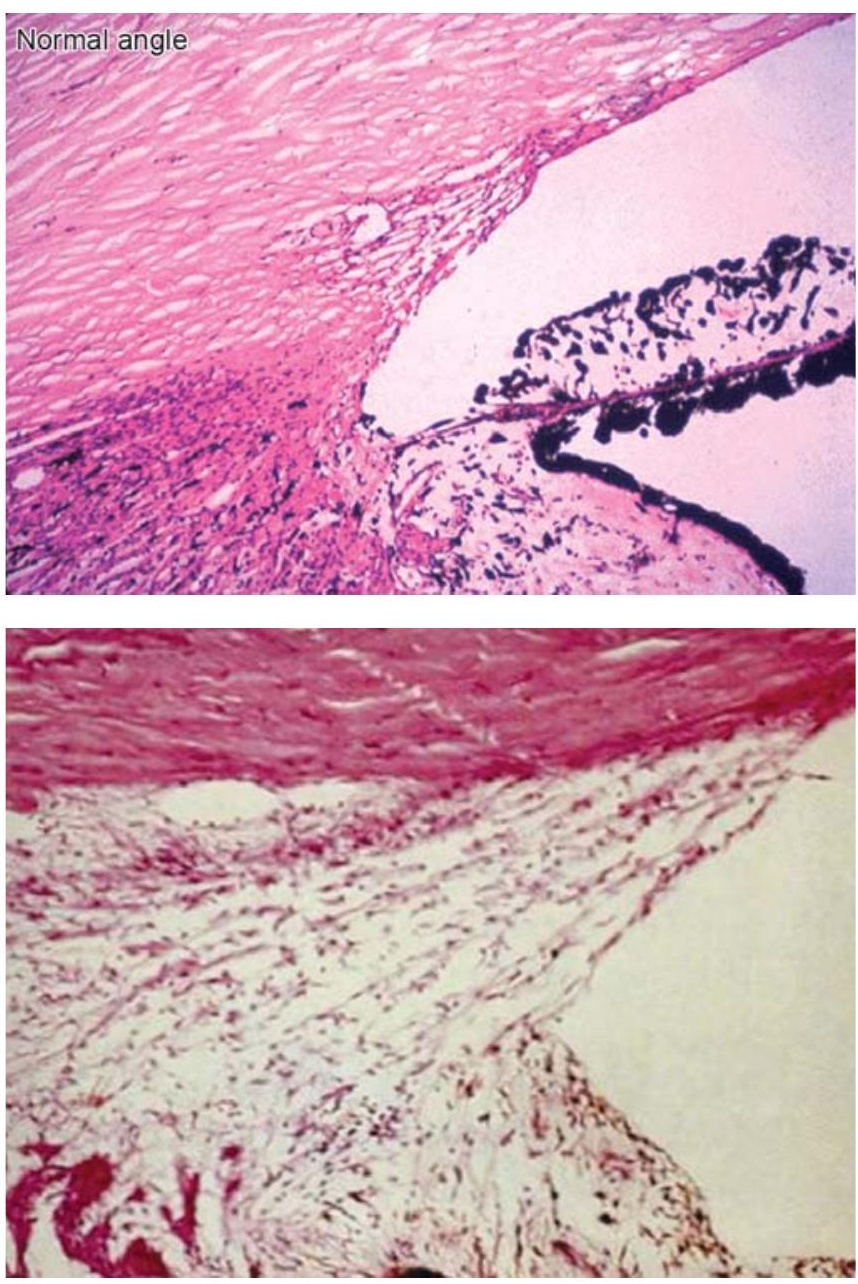

Fig. 1: Contrast the normal well-developed anterior chamber angle (above) as compared to the angle of the anterior chamber in an infant with primary congenital glaucoma (below). The features in the angle of congenital glaucoma include anterior insertion of iris, rudimentary scleral spur, insertion of ciliary muscle directly into the trabecular meshwork and undifferentiated trabecular meshwork. (Tripathi \& Tripathi, Embryology of the anterior segment of the human eye)

usually manifested at birth or within three years of life. The incidence of primary congenital glaucoma in the west is estimated to be about one in 10-15,000 live births, but is likely to more common in the developing world like India, due to increased consanguinity among many ethnic groups in these populations. A population based assessment of childhood blindness in southern India has estimated the incidence to be about one in 3300 in the Indian state of Andhra Pradesh. While $60 \%$ children with congenital glaucoma present by 6 months of age, a majority (80\%) are diagnosed by the first year of life. Males predominate in a ratio of 3:2, while the involvement is bilateral in about 70$80 \%$. Most cases of primary congenital glaucoma are sporadic, while children with familial glaucoma reveal an autosomal recessive pattern with variable and incomplete penetrance. Autosomal dominant pattern of inheritance is also reported in some pedigrees. Genetic studies have revealed that mutations in the CYP1B1 gene encoding the cytochrome P450 enzyme in what is known as the GLC3A locus of the chromosome 2p21 are associated with primary congenital glaucoma ${ }^{5}$.

Recent studies have focused on the localization of this enzyme in this eye and its possible functions related to ocular development and possible molecular mechanisms by which the mutation can cause glaucoma. The generic defect is not totally penetrant which in other words means that there are people who do show the mutated gene type but do not have disease at birth or in infancy. This has suggested the possibility of a modifier gene which might modify the activity of CYP1B1. Recently a paper suggesting that a possible modifier may exist has been published. In the CYP1B1 knockout mouse it was observed that the phenotype was expressed only in tyrosinase deficient mice. $^{11}$

More recently Deepak Edward et al (personal communication) using immunohistochemistry have identified that CYP1B1 in fetal eyes and in adult eyes is mainly localized to the ciliary epithelium in the anterior segment and with very little expression in the trabecular meshwork. The expression in the ciliary epithelium appears to be confined to the non-pigmented ciliary epithelium. Based on this it has been hypothesized that CYP1B1 through an indirect mechanism or possibly through a chemical messenger affects the normal development of the trabecular meshwork leading to obstruction of aqueous humor outflow. This gene product is likely a substrate of the enzyme CYP1B1 and might be a protein product/cytokine present in the aqueous humor. Further studies are required, however, to substantiate the presence of these proteins in the aqueous humor of children with congenital glaucoma.

Ocular enlargement occurs due to the fact that the infant's eye is largely distensible since the corneal and scleral collagen are not mature enough to resist distention with increased IOP. The changes primarily affect the cornea, sclera, corneoscleral limbus, the optic nerve, scleral canal and the lamina cribrosa. Corneal enlargement is a very specific sign of congenital glaucoma. The normal neonatal horizontal corneal diameter at birth is $10-10.5 \mathrm{~mm}$ at birth and increases by about 0.5 to $1.0 \mathrm{~mm}$ in the first year of life. A horizontal corneal diameter of $12 \mathrm{~mm}$ in the first year of life associated with corneal edema is pathognomonic of glaucoma (differential diagnosismegalocornea but the cornea is clear in this condition ). Asymmetrical enlargement of cornea is more easily diagnosed and is characteristic of congenital glaucoma. Corneal enlargement from elevated IOP is remarkable in the first three years of life though the sclera is deformable until about ten years of age (Figs 2 to 4 ). 


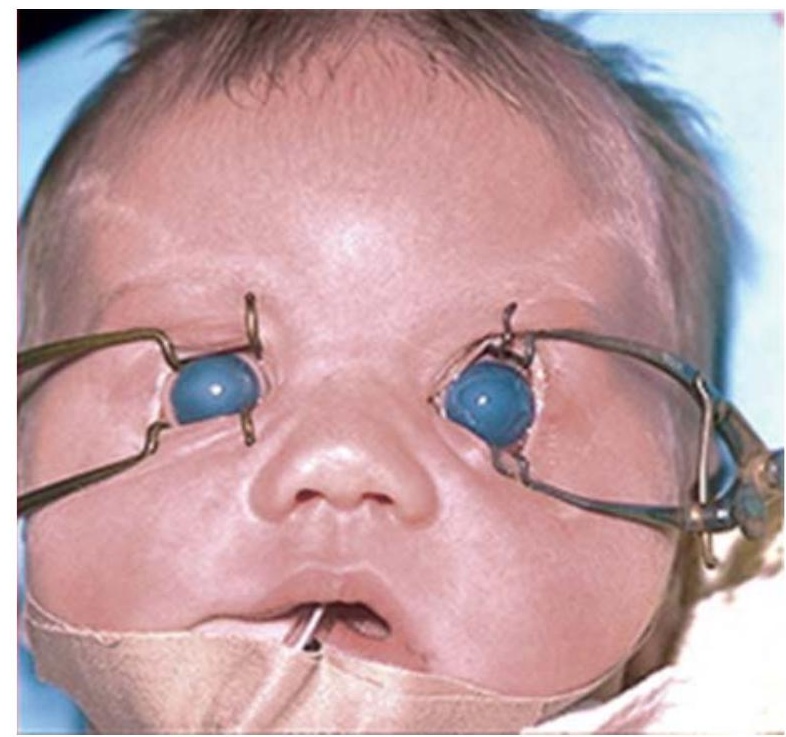

Fig. 2: An infant with features typical of primary congenital glaucomaObserve asymmetrical corneal enlargement and corneal edema
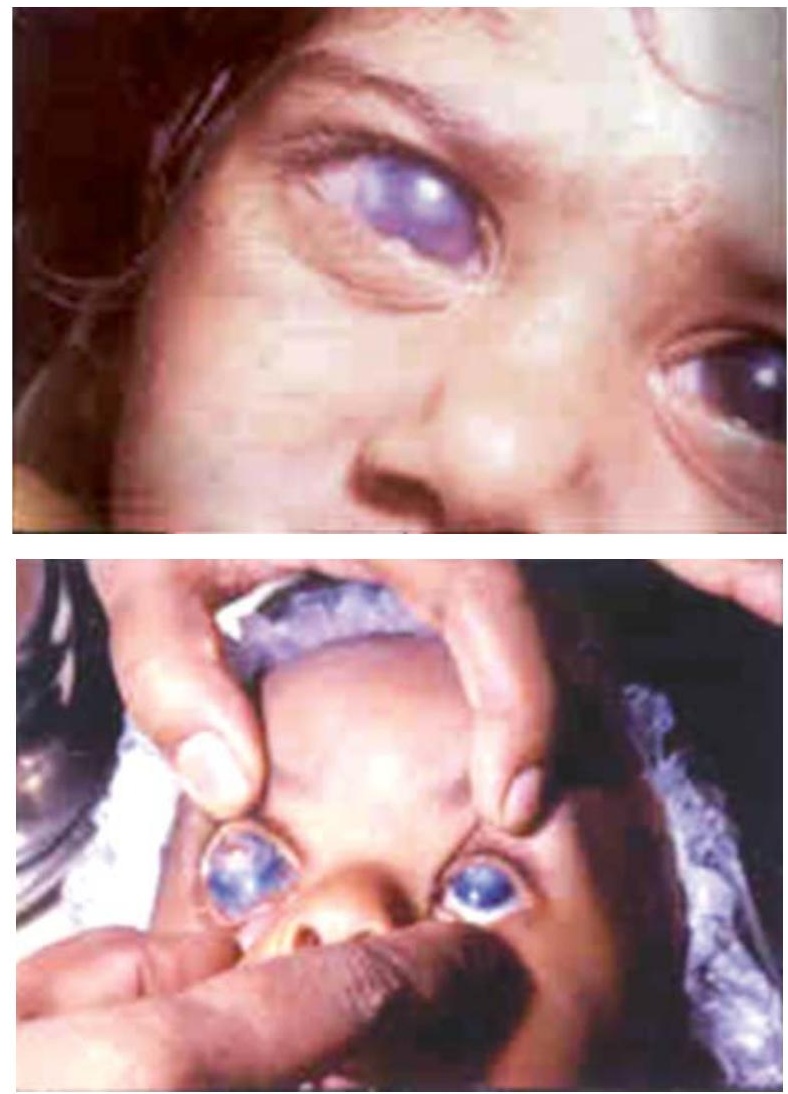

Fig. 3: Children with advanced congenital glaucoma. Observe buphthalmos with corneas exceeding $15 \mathrm{~mm}$ in diameter and corneal scarring. Children presenting with such late manifestations have poor visual prognosis to surgical or medical treatment. Early diagnosis and surgical correction of underlying pathophysiology is the key to success of treatment in these children

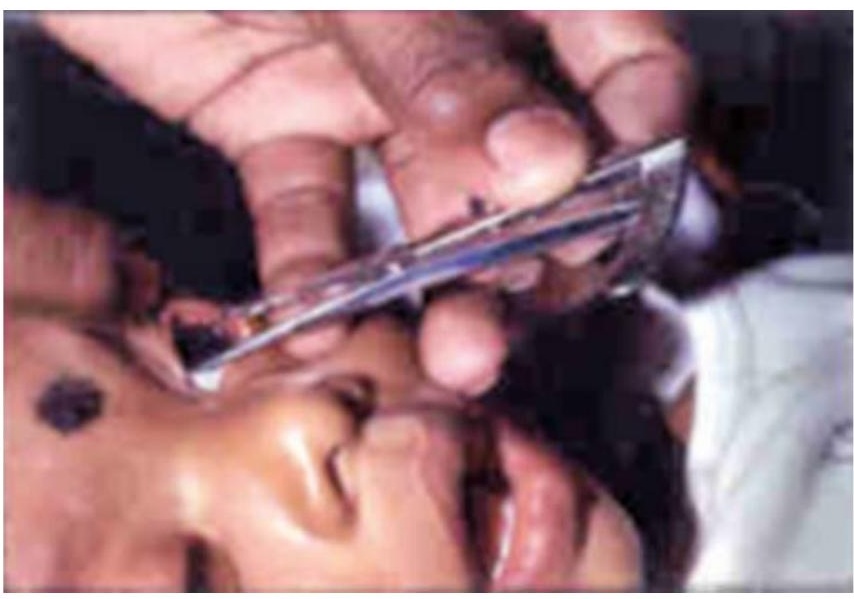

Fig. 4: Estimation of the horizontal corneal diameter is an essential tool in diagnosis of congenital glaucoma. A measurement exceeding $12 \mathrm{~mm}$ in a child is pathognomonic of glaucoma, provided other causes of megalocornea can be excluded

\section{Clinical Features}

History and Symptomatology

External Examiantion

Corneal diameter

Corneal edema

IOP elevation (tonometry)

Refractive error and amblyopia

Slit lamp evaluation and gonioscopy

Optic nerve examination

\section{History and Symptomatology}

Epiphora, photophobia and blepharospasm represent the classic triad of symptoms of congenital glaucoma. Any combination of these symptoms should arouse the suspicion of glaucoma in a child. These symptoms are a result of corneal irritation from corneal epithelial edema associated with elevated intraocular pressure (IOP). Infantile glaucomas may also present as a red eye mimicking conjunctivitis and resulting in a delay in diagnosis. Elevated IOP results in ocular enlargement with maximal enlargement occurring at the corneoscleral junction. Loss in corneal transparency with corneal haze may be initially intermittent and precede breaks in descemet membrane.

\section{EXTERNAL EXAMINATION AND SLITLAMP BIOMICROSCOPY}

Haab's striae ${ }^{6}$ are breaks in descemet membrane as increased IOP stretches the corneal endothelium and the Descemet's membrane and are characteristic of congenital glaucoma with onset before the age of three and in corneas with diameter exceeding $12.5 \mathrm{~mm}$ (Fig. 5). The edge of the rupture in descemet membrane contracts into ridges and scrolls, penetration of 

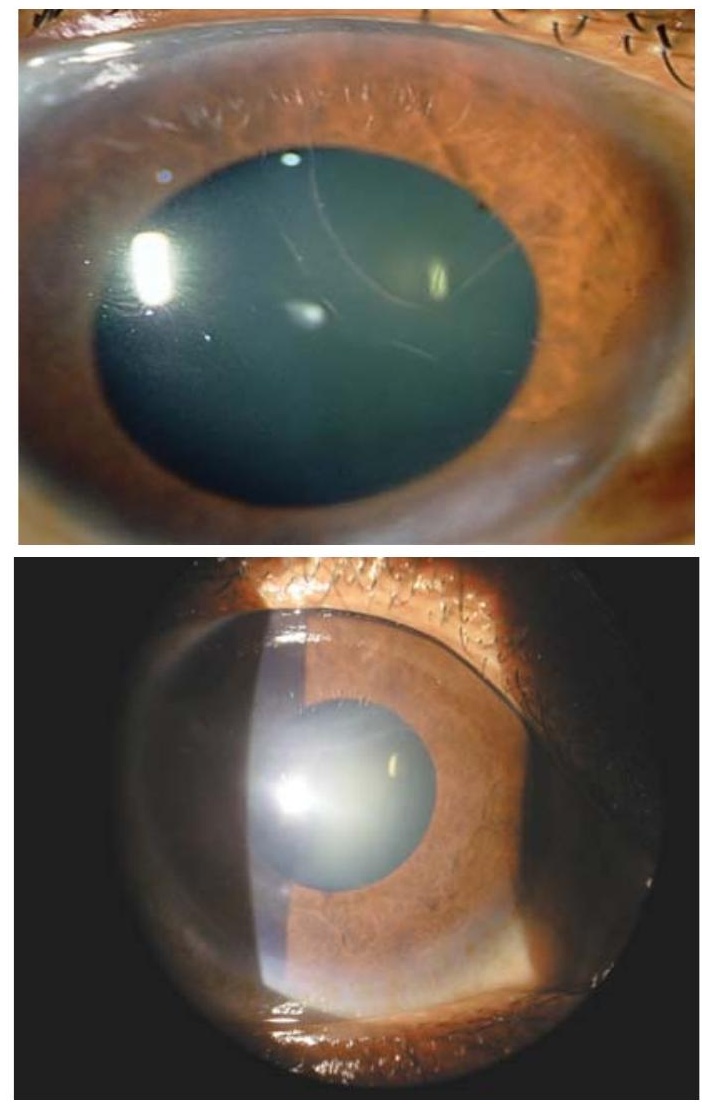

Fig. 5: Haab's striae typically seen in a child with megalocornea and primary congenital glaucoma.

aqueous compounds any diffuse corneal edema. Haab’s striae result from new basement membrane laid down by the endothelial cells. These striae are horizontal and linear when seen centrally and curvilinear and vertical when they are peripheral and parallel the limbus. As IOP normalizes and corneal edema clears,endothelial overgrowth repairs the tears but linear striae persist. Reduced endothelial count in such eyes may be evident on specular microscopy. Persistence of elevated IOP in unresolved congenital glaucoma causes stromal corneal edema, corneal scarring and irregular corneal astigmatism.

Distention of sclera under the influence of elevated IOP causes scleral thinning with blue sclera with increased visibility of the underlying uvea, increased axial length and axial myopia and buphthalmos. Unilateral or asymmetric congenital glaucoma result in myopic astigmatism, anisometropia and amblyopia. Enlargement of the globe under the influence of IOP elevation in the first three years of life causes a myopic shift in the refractive status of the eye. Astigmatism is commonly owing to Haab's striae and amblyopia is the rule in asymmetricdal congenital glaucoma. Children between 3-10 years age with glaucoma develop progressive myopia under the influence of elevated IOP due to scleral distension and stretching although they exhibit no corneal enlargement. Loss of hyperopia in aphakic children is evidence of glaucoma in aphakia.

\section{IOP and Tonometry}

The most accurate measurements of IOP are those obtained in an awake and cooperative child under topical anesthesia and blepharospasm or a struggling/crying child can artifactually elevate IOP. Sedatives and anesthetics alter IOP to a varying extent. While most inhalational anesthetics like halothane lower IOP, succinylcholine and ketamine elevate IOP. Most reliable IOP are obtained under intramuscular ketamine and in the lighter planes of inhalational anesthetics prior to intubation. Perkins tonometer and tonopen are reliably used to measure IOP in children. Infants and Ilyoung children appear to have IOP lower than those expected in adults: Mean IOP of $9.59 \mathrm{mmHg}$ was found in the newborn which had risen to 13.95 by 7 or 8 years of age. Infants with primary congenital glaucoma usually present with IOP exceeding 30-40 mmHg when unanesthetised, but may be much lower under the influence of inhalational anesthetics.

\section{Gonioscopic Evaluation of the Anterior Chamber Angle in Primary Congenital Glaucoma}

Evaluation of the anterior chamber angle aids in diagnosis of primary congenital glaucoma and helps in differentiating this condition from other entities with similar clinical features. Ideally a Koeppe’s gonio lens with a portable slit lamp delivery system is utilized for the purpose. A Goldmann gonio lens is also used for viewing the angle through the operating microscope. Corneal clouding may obscure the details of the angle which may be improved by using topical glycerine or 70\% alcohol with a cotton applicator. Edematous epithelium may also be removed using a surgical blade to improve visualization of the angle and the anterior chamber.

The anterior chamber angle in infants vastly differs from that of adults. In the normal eye of the newborn, the iris usually inserts posterior to the scleral spur. The iris insertion into the angle is flat and the angle recess is not formed. The trabecular meshwork appears thicker and more translucent than that of an adult. Pigmentation is absent unlike in adults. Gonioscopy of the eyes in infants with primary congenital glaucoma reveals anterior insertion of the iris directly into the trabecular meshwork. The surface of the trabecular meshwork has a stippled appearance and the meshwork appears thicker than normal. The peripheral iris shows thinning of the anterior stroma. The angle is usually devoid of vessels, although loops of vessels from the major arterial circle is seen above the iris surface and has been referred to as the Loch Ness Monster phenomenon. The peripheral iris inserting into the trabecular meshwork may appear translucent and is often referred to as the Lister's morning mist. 


\section{Optic Nerve Changes in Congenital Glaucoma}

Optic nerve cupping occurs rapidly in infants with elevated IOP and unlike in adult eyes is also rapidly reversible with normalization of $\mathrm{IOP}^{7}$. Reversibility of optic nerve cupping with treatment in congenital glaucoma appears to be due to incomplete development of the connective tissue comprising the lamina cribrosa with posterior movement of the lamina and enlargement of the scleral canal and consequently of the cup to disc ratio in response to IOP elevation, and the more resilient connective tissue in the optic nerve head cause reversal of cupping with normalized IOP. Persistent IOP elevation, however, causes glaucomatous optic atrophy due to loss of ganglion cells.

Uncontrolled and refractory IOP elevation in congenital glaucoma causes corneal scarring and ulceration and perforation with continued enlargement of the cornea and the globe. Stretching and rupture of zonules causes lens subluxation; hyphema, retinal detachment and pthisis bulbi are often the final outcome of untreated or refractory glaucomas in infancy and childhood.

Ultrasonic ocular biometry aids in diagnosis and follow up of children with congenital glaucoma. Ultrasonic measurements of the axial length of the eye is a valuable tool to diagnose congenital glaucomas. The anterior chamber depth and axial length are significantly increased while the thickness of the lens is decreased. The reduction in lens thickness and corneal flattening limits the myopic shift seen with increasing axial length of the eye with progressive glaucoma.

\section{Differential Diagnosis of Primary Congenital Glaucoma}

Although a child with corneal and ocular enlargement and corneal haze due to edema presenting with blepharospasm, photophobia and tearing typically has primary congenital glaucoma, it is essential to exclude other childhood eye diseases with overlapping signs and symptoms simulating congential glaucoma.

Nasolcacrimal duct obstruction in the newborn and infants is associated with tearing, although photophobia and other corneal changes typical of congenital glaucoma is absent. Obstruction of nasolacrimal passage is also associated with fullness in the region of the lacrimal sac and a chronic mucopurulent discharge. Redness and tearing can be a manisfestation of any of the several causes of conjunctivitis in children, including bacterial, viral and chlamydial, including the chemical conjunctivitis as a result of silver nitrate prophylaxis. Corneal epithelial defects and abrasions are frequent causes of acute ocular irritation, tearing and photophobia.

Corneal dystrophies (Meesman's and Reis Bucklers) which present in the early years of life may also manisfest with ocular irritation and tearing and need to be differentiated from congenital glaucoma. Congenital hereditary endothelial and stromal dystrophy also manifest with corneal edema, and tearing differentiated due to absence of corneal enlargement. Inflammatory keratitis and iridocyclitis occasionally cause corneal clouding and edema associated with pain and tearing. Rubella keratitis typically causes corneal clouding and enlargement even in the absence of secondary glaucoma and is difficult to be differentiated from congenital glaucoma. The fact that secondary glaucoma due to abnormal anterior chamber development commonly accompanies Congenital Rubella Syndrome further compounds the problem of accurate diagnosis and appropriate treatment. Characteristically, when rubella viremia is present in the third trimester of pregnancy, anomalous anterior chamber angle development results in glaucoma with no other features of the maternal rubella syndrome.

Extreme degrees of axial myopia can present with enlarged globes and large corneas, but features of posterior pole such as tilted optic discs, peripapillary myopic crescent and choroidal mottling which are not characteristic of congenital glaucoma serve to distinguish the two conditions. Megalocornea is a condition characterized by large corneas, often upto 14-16 mm in diameter. Features of congenital glaucoma, such as corneal edema and descemet membrane breaks, elevated IOP and optic nerve cupping are absent. The condition appears to have sex linked inheritance with $90 \%$ reported in males and families have been studied in which some members have megalocornea and others, primary congenital glaucoma. Some investigators consider megalocornea to be a forme fruste of primary congenital glaucoma, and hence individuals with large corneas need to be followed up for life to exclude glaucoma.

In sclerocornea, the opaque corneal tissue extends onto the cornea. Obstetric trauma can cause rupture of the descemet membrane with resultant corneal edema and clouding. The breaks in birth trauma are usually oriented vertically, although exceptions do occur with the breaks being distributed in a horizontal or curvilinear fashion. Birth trauma is unilateral, with no corneal enlargement or elevated IOP and associated with periorbital skin changes like bruising when examined immediately after birth. The left eye is more often involved since the left anterior occiput is the most common presentation of the infant's head.

Several inborn errors of metabolism cause corneal clouding and mimic congenital glaucoma. Mucopolysaccharidoses are characterized by excessive storage of mucopolysaccharides due to their defective degradation from lack of the lysosomal acid hydrolases- examples include Hurler's Syndrome, Scheie Syndrome, Morquio Syndrome and the Maroteaux-Lamy Syndrome. Cystinosis is due to defective lysosomal transport system with deposition of cystine crystals in cornea presenting with intense photophobia; with renal failure and salt-pepper retinopathy in the nephropathic type cystinosis. Mucolipidosis, sphingolipidoses, metachromatic leucodystrophy, glucose 6 
phosphate deficiency are other storage diseases associated with corneal clouding, but differentiated from congenital glaucoma by absence of elevation of IOP and corneal enlargement.

Congenital Hereditary Endothelial Dystrophy (CHED) is an important condition that is mistaken for congenital glaucoma, since the condition is manifest at birth or within first two years of life. It is inherited both as autosomal recessive and dominant condition with variable expressivity with mild posterior corneal changes to diffuse corneal edema. The cornea is thickened to three times normal, often over-estimating the IOP measured by applanation tonometry, but corneal enlargement is typically absent. Corneal clouding is often symmetrical with no descemet breaks or corneal scarring. Although some authors report tearing and photophobia in children with CHED, this is extremely uncommon in the authors' clinical experience and the absence of tearing, photophobia, blepharospasm associated with absence of corneal enlargement is considered typical of CHED and helps differentiate from congenital glaucoma. The distinction, nevertheless is crucial, since a diagnosis of congenital glaucoma necessitates immediate surgical intervention to preserve visual function. In the instance of difficulty in differential diagnosis, especially in the first few years of life, it is prudent to periodically evaluate these children, especially to exclude corneal or ocular enlargement ( by ultrasonography to exclude axial myopia). It is, however, not unusual to encounter congenital glaucoma associated with CHED and in these eyes, corneal edema persist after normalization of IOP following surgical intervention. The precise diagnosis, however, is established only after the histopathological examination of the corneal buttons obtained after penetrating keratoplasty in these eyes.

\section{Management}

Early detection and appropriate treatment of primary congenital glaucoma cannot be overemphasised, since neglect and inappropriate treatment can cause profound visual loss in the children with increase in the blind-years. With improvement in the understanding of genetics and pathophysiology of the disease and improvised surgical techniques, it is often possible to prevent the children going blind, with preservation of useful vision. The principal aim in the management of children with congenital glaucoma is preservation of visual function, ocular structural integrity, promotion of development of binocular stereoscopic vision and early visual rehabilitation to prevent amblyopia.

Primary congenital glaucoma is an essentially surgical disease and medical treatment is used only as a temporizing measure or as a supplement to surgical intervention when IOP remains difficult to be controlled. Lasers also have only a supportive role. Several factors, including the structural defects characterizing glaucoma, age at presentation, corneal clarity and enlargement, associated systemic features and severity of glaucoma influence the ophthalmologists' decision on the type of treatment necessitated. It is important to discuss the prognosis with the parents and ensure they remain committed to the child's prolonged treatment.

\section{MEDICAL THERAPY FOR PRIMARY CONGENITAL GLAUCOMA}

Since primary congenital glaucoma is essentially characterized by elevation of IOP due to a structural defect, medical therapy plays little role in primary therapy of glaucoma and the treatment is essentially surgical to overcome the developmental defect causing resistance to aqueous outflow. However, drugs to reduce IOP may be used to supplement surgical treatment when intraocular pressure reduction is considered inadequate or when surgery fails to adequately contain the pressure elevation. There are no randomized controlled clinical trials available on the efficacy of pressure lowering drugs in children and the available data of safety and efficacy are from studies conducted in adults and these drugs need to be used in children with caution, especially in those with systemic diseases; and carefully evaluate the risk and benefits of individual therapeutic agents and use the minimal effective dose of the drugs possible to achieve the maximal pressure lowering effect, while monitoring the children for ocular and systemic side effects.

$\beta$ Blockers: Timolol is the most widely used beta blocker in reduction of intraocular pressure in children, but various studies that have evaluated timolol either as a single or adjunctive drug have reported adequate IOP reduction in only a third. Plasma timolol levels in children, particularly in infants, after treatment with $0.25 \%$ timolol significantly exceed that seen in adults after instillation of $0.5 \%$ timolol. Increased concentration of plasma timolol in children explained by the smaller volume of distribution of the drug as compared to adults and hence an increased likelihood of systemic adverse effects in the young. Reduction in heart rate, exacerbation of asthma and apnoea have been reported, necessitating withdrawal of the drug due to adverse effects in $4-13 \%$ of children treated. A detailed systemic evaluation to exclude pulmonary and cardiac diseases is to be performed prior to administration of this drug in children and infants and needs to be avoided in neonates and the premature due to risk of sleep apnoea. When indicated, timolol gel forming solutions may be preferred, since this formulation is expected to reduce the adverse effects of the active drug due to lesser systemic absorption.

Carbonic Anhydrase Inhibitors: Systemic CAI, such as acetazolamide have been recommended in the dosage of 5-10 $\mathrm{mg} / \mathrm{kg} /$ day in divided doses as a temporizing measure to reduce IOP and corneal edema prior to surgery. Prolonged treatment with acetazolamide is associated with growth suppression and metabolic acidosis in children. They are also subject to other serious adverse effects like drug idiosyncracy and bone marrow 
suppression and prolonged therapy with oral acetazolamide is best avoided. Topical CAI, as $2 \%$ dorzolamide, effectively reduces IOP in children, though the IOP lowering potency is studied to be lowered as compared to oral acetazolamide. Topical dorzolamide is currently the preferred IOP lowering agent of choice in infants and children and may administered two or three times daily, since the drug is devoid of serious systemic adverse effects of timolol and oral acetazolamide. In older children with no contraindications, fixed combination therapy of timolol and dorzolamide may be used, which is convenient and gives the advantage of use of employing two drugs while simplifying the dosage schedule.

Prostaglandin Analogues: The use of prostaglandins and their efficacy in children with congenital glaucoma is not adequately studied. Even in older children with Struge Weber suyndrome, only a third of patients treated with latanoprost responded favorably with therapy. Use of latanoprost or any of the prostaglandin analogues in childhood glaucomas is currently not recommended.

Alpha Receptor Agonists are an important adjunct in treatment of adult glaucomas. Specific Alpha ${ }^{2}$ receptor agonists like brimonidine is widely used in management of chronic glaucomas. However, brimonidine easily crosses the immature blood brain barrier of children and causes adverse central nervous system effects like drowsiness and even respiratory depression. Apart from extreme fatigue, infants on brimonidine have developed recurrent episodes of unresponsiveness, hypotension, hypotonia, hypothermia and bradycardia. Failure of recovery from anesthesia and death of premature infants have been attributed to use of brimonidine. Use of brimonidine and other alpha receptor agonists are not recommended in children younger than eighteen years.

Cholinergic drugs ( Pilocarpine) do not seem to have a useful role in medical treatment of congenital glaucoma. Although miotics increase facility of outflow facility and reduce IOP in normal and open angles, they do not seem to be effective in eyes with congenital glaucoma with an abnormally developed angle with anterior insertion of the ciliary musculature into the trabecular meshwork. Pilocarpine, may however, be useful in children with glaucoma in aphakia and pseudophakia with open iridocorneal angles.

\section{SURGICAL TREATMENT OF PRIMARY CONGENITAL GLAUCOMA}

Early detection and surgical treatment is of prime importance in ensuring IOP reduction and visual preservation in children with congenital glaucoma. In eyes with early congenital glaucoma with corneal edema and minimal ocular and corneal enlargement, goniotomy is the initial procedure of choice. However, in India and other developing nations most children with congenital glaucoma report with corneal enlargement and scarring and goniotomy is usually difficult owing to poor visualization of the angle structures and need ab externo trabeculotomy, often combined with trabeculectomy.

\section{Goniotomy}

Goniotomy is considered as the treatment of choice by many surgeons in primary congenital glaucoma, provided the cornea allows satisfactory visualization of angle. The procedure, initially practiced by Barkan ${ }^{8}$, aims to remove the obstructing tissue in the angle that causes resistance to aqueous flow (Fig. 6).
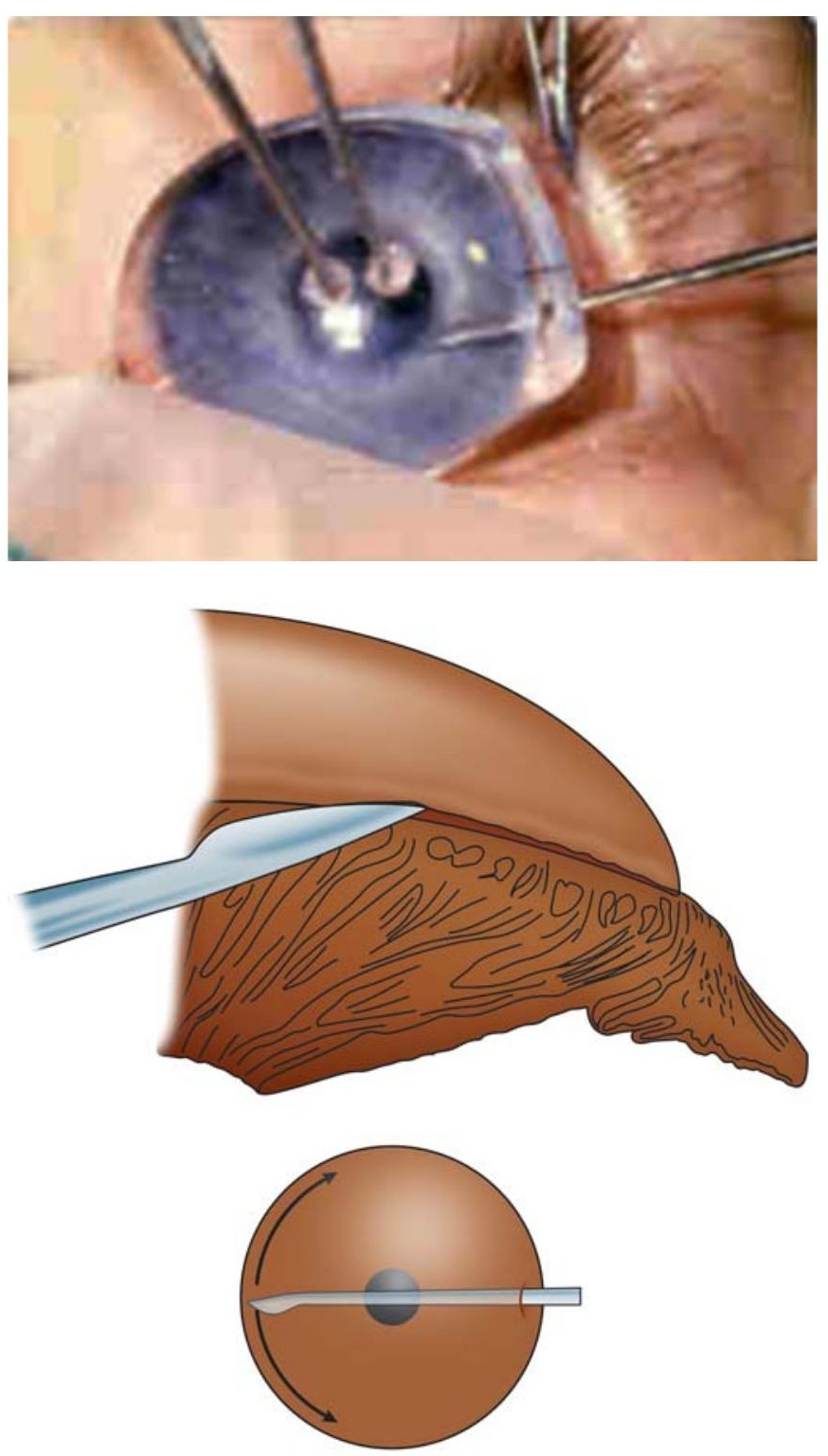

Fig. 6: The goniotomy procedure in progress 


\section{Procedure}

Corneal edema may be cleared by the use of $20 \%$ glycerol drops immediately before surgery; if unsuccessful, epithelial debridement with absolute alcohol provides adequate view of the angle to allow goniotomy in most of the cases.

- $\quad$ To be performed safely - general anesthesia, on operating microscope, a contact lens (e.g. Barkam lens) and a tapered goniotomy blade are required.

- Preoperatively pilocarpine helps to open the angle and protects the lens by constricting the pupil.

- The inner portion of the nasal trabecular meshwork over 90-120 degree is incised with the goniotomy bade under direct visualization of the angle with the help of gonio lens.

- A mild hyphema on withdrawal of the knife from the anterior chamber is typical and indicates a correctly placed incision.

- Goniotomy can be repeated in the event of unsuccessful IOP control preferably in the temporal quadrant.

- Goniotomy presumably relieves the compressive traction of anterior uvea on the meshwork and eliminates any resistance imposed by incompletely developed inner meshwork.

- Advantages of Goniotomy: Less traumatic and safe in experienced hands, rapid, can be repeated and spares the conjunctiva for possible later surgery.

- Disadvantages: Procedure not possible if details of angle structure are not clear, technically demanding requires special instruments, needs experienced surgeons and possibility of corneal endothelial, angle and lens trauma.

- $\quad$ Success rate following multiple goniotonies in 70-90\% with medium term follow up.

- Moor fields experience showed a 20\% relapse rate over a 30 years-period with no peak age of relapse emphasizing the importance of life-long follow-up.

- Infants presenting between 2 to 8 months have best prognosis and surgery becomes less effective with increasing age. The worst prognosis occurs for infants with elevated pressures and cloudy corneas at birth.

\section{TRABECULOTOMY ${ }^{9}$}

It is the procedure of choice by many surgeons particularly when cornea is opaque. Corneal clarity is not a pre- requisite since the procedure is ab externo and identifies the schlemm's canal by external approach. Results of trabeculotomy in several studies have reported to be as favorable as initial goniotomy procedures.

\section{Procedure}

A limbal or fornix based conjunctival flap is dissected. A trabeculectomy scleral flap is fashioned and Schlemm's canal located by slowly deepening a $2 \mathrm{~mm}$ radial incision placed at the corneal scleral junction. An accurate knowledge of the surgical anatomy of the limbus is essential to identify the Schlemm's canal. The junction of the blue white sclera marks the location of the scleral spur and the Schlemm's canal is a mm anterior to this structure. The trabeculotome is gently threaded into the canal and swept into the anterior chamber, rupturing the internal wall of Schlemm's canal and trabecular meshwork and directly exposing it to aqueous humor. The procedure is repeated to other side of the canal. The scleral flap is tightly closed. Accurate localization of Schlemm's canal is the most important step. Schlemm's canal is not found in $4-20 \%$ of the cases. A mild to moderate hyphema is a regular occurrence, and often confirms accurate identification of the Schlemm's canal. Appearance of aqueous as the lumen of the canal is opened is also evidence of entry into the canal of Sclemm.

Mendincino et al have described a 360-degree suture trabeculotomy using 6/0 polypropylene which is threaded into entire Schlemm's canal from 1 or 2 cut down sites and when the suture is pulled towards the entry of the canal in the surgical limbus, the entire lumen of the canal is opened into the anterior chamber.

\section{Advantages}

The procedure can be performed in opaque corneas, many components of technique similar to trabeculectomyand higher success rate has been reported by some studies when trabeculotomy is combined with Trabeculectomy. Even if one fails to accurately identify the Schlemm's canal, the procedure could be completed by proceeding with trabeculectomy as in adult glaucomas.

\section{Disadvantages}

Angle structures are not directly visualized in trabeculotomy hence complications though seldom serious may be more frequent than goniotomy. Potential complications include descemets membrane stripping, iris prolopse, iridodialysis, cyclodialysis with persistent hypotony, false passages, lens subluxation and a prolonged flat anterior chamber. It also damages conjunctiva and prejudices success of future filtering surgery.

Primary trabeculectomy is also an optional surgical procedure in eyes with congenital glaucoma and most ophthalmologists are familiar with the technique. The procedure is also easier to perform than goniotomy or trabeculotomy. Several published reports have documented comparable results with primnary trabeculectomy as compared to goniotomy or trabeculotomy. In addition, a combined trabeculotomy and trabeculectomy may be performed as a primary or secondary procedure in eyes that are likely to have poorer surgical outcome following trabeculotomy or goniotomy owing to advanced nature of the disease. A higher incidence of successful intraocular pressure control with primary combined 
trabeculotomy and trabeculectomy has been reported in some ethnic groups, especially in the developing world like India, ${ }^{10}$ where children present late with advanced disease and buphthalmos (Fig. 7). In eyes with refractory congenital glaucoma, trabeculectomy with antimetabolites, glaucoma drainage devices and laser cyclodestructive procedures are attempted.

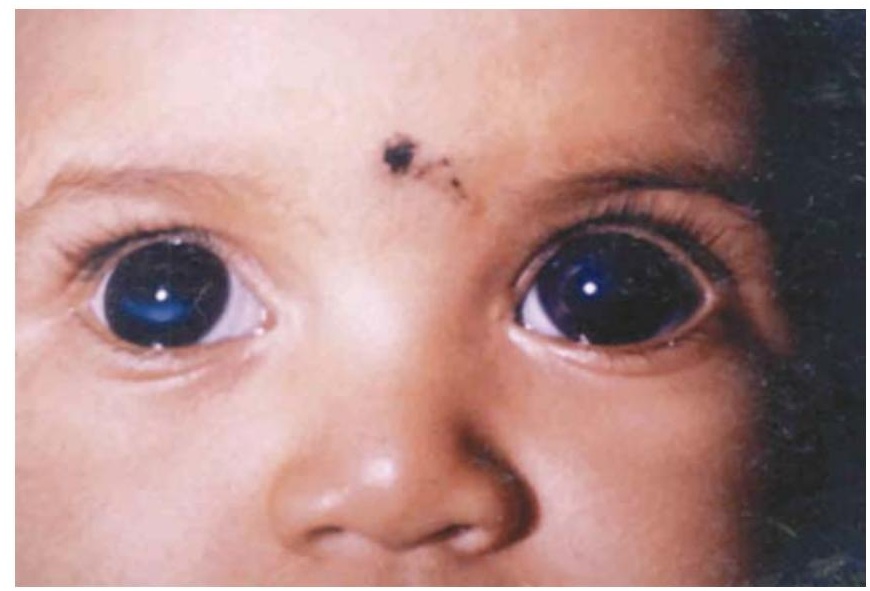

Fig. 7: A child with adequate IOP control following combined trabeculotomy with trabeculectomy. Asymmetric corneal enlargement and corneal scarring inferior to the visual axis in the right eye can still be seen. Such children often have productive vision with visual rehabilitation and treatment for amblyopia, which is an essential component of integral treatment of congenital glaucoma

\section{REFERENCES}

1. Shaffer RN, Weiss DI. Congenital and Paedriatic glaucomas. CV Mosby: St Louis, 1970.

2. Barkan O. Pathogenesis of congenital laucoma. Gonioscopic and anatomic observation of the angle of the anterior chamber in the normal eye and in congenital glaucoma. Am J Ophthalmol 1955;40:1-11.

3. Maumenee AE. The pathogenesis of congenital glaucoma. A New Theory. Trans Am Ophthalmol Soc 1958;56:507-70.
4. DeLouise VP, Anderson DR. Primary infantile glaucoma. Surv Ophthalmol 1983;28:1-19.

5. Stoilov I, Akarsu AN, Sarfrazi M. Identification of three different truncating mutations in cytochrome P4501b1 (CYP1B1) as the principal cause of primary congenital glaucoma in families linked to the GLC3Alocus on chromosome 2p21. Hum Mol Genet 1997,6:641-47.

6. Waring GO, Laibson PR, Rodriguez, M. Clinical and pathological alteration of Descemet membrane with emphasis on endothelial meaplasia. Surv Ophthalmol 1974;18:325-68.

7. Quigley HA. The pathogenesis of reversible cupping in congenital glaucoma. Am J Ophthalmol 1977;84:358-70.

8. Barkan O.Surgery for congenital glaucoma. Review of 196 eyes operated by goniotomy. Am J Ophthalmol 1953;36:1523-34.

9. Burian HM, Allen L. Trabeculotomy ab externo, a new glaucoma operation: Technique and results of experimental surgery. Am J Ophhtalmol 1962; 53: 19-26.

10. Mandal AK, Bhatia PG,Bhaskar A, Nutheti R. Long term surgical and visual outcomes in Indian children with developmental glaucoma operated within six months of birth. Ophthalmology 2004; 111: 283-90.

11. Libby RT, Smith RS, Savinova OV, Zabaleta A, Martin JE, Gonzalez FJ, John S. Modification of ocular defects in mouse developmental glaucoma models by tyrosinase. Science. 2003 Mar 7; 299(5612): 1578-81.

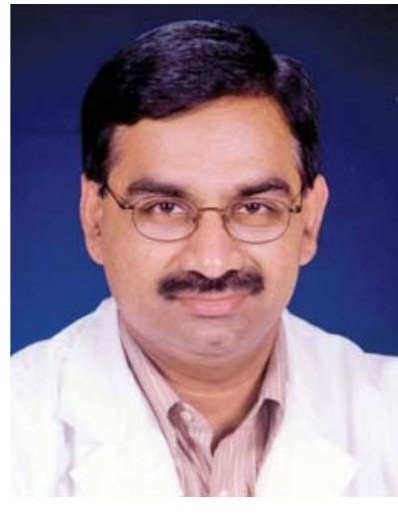

R Krishnadas

(krishnadas@aravind.org)

"God grant me the serenity to accept the things that I cannot change, the courage to change the things that I can, and the wisdom to know the difference" 